\title{
Granulocytic Sarcoma: A Report of Four Cases Diagnosed on FNAC
}

\author{
Sunaina Agarwal ${ }^{1 *}$, Abhay Agarwal ${ }^{2}$, Rimi Pandey ${ }^{3}$ and Anshu Sharma ${ }^{1}$ \\ ${ }^{1}$ Pathology department,Kasturba Medical college,Manipal,India \\ ${ }^{2}$ Surgery department, Vivekananda Polyclinic \& Institute of Medical Sciences, Lucknow,India. \\ ${ }^{3}$ Pathology department, Vivekananda Polyclinic \& Institute of Medical Sciences, Lucknow,India
}

\section{ABSTRACT}

The term "granulocytic sarcoma" refers to an extramedullary collection of immature myeloid cells; rarely,it may precede peripheral blood or bone marrow involvement, presenting as a diagnostic challenge. The present communication deals with four cases of granulocytic sarcoma. Fine needle aspiration cytology (FNAC) was performed for all the cases and a diagnosis of granulocytic sarcoma was suspected. Subsequently, a complete blood count was performed which revealed blasts in the peripheral blood and a diagnosis of acute myeloid leukemia (AML) was confirmed on bone marrow examination. Here, we emphasize the utility of FNAC for making the correct diagnosis of this entity in cases where AML was previously not suspected.

\section{Keywords: AML; Auer rod; FNAC; Granulocytic Sarcoma.}

\section{Introduction}

Granulocytic sarcoma (GS) or myeloid sarcoma is a tumoral lesion which consists of immature cells of granulocytic series. The term chloroma was assigned to this condition due to its green colour attributed to the enzyme myeloperoxidase (MPO).It has been identified as an extramedullary presentation of acute leukemias especially in acute myeloid leukemia. Less commonly, it has been observed during the course of myelodysplastic syndrome, chronic myeloid leukemia and other myeloproliferative diseases. ${ }^{[1]} \mathrm{GS}$ may precede AML where bone marrow aspiration and biopsy might not reveal any haematological disease; this type of MS is called isolated, primary or nonleukemic MS.It can occur as solitary or multiple. It is a rare disease with an incidence of 2/1.000.000 in adults. Very few cases have been reported in the literature so far. ${ }^{[2]} \mathrm{GS}$ can occur at any age, both paediatric and elderly population can be affected. ${ }^{[2]}$ It can involve any site of the body but has more predilectionfor soft tissues, bone, peritoneum, lymph nodes and gastrointestinal system. ${ }^{[3]}$ Other rare sites that have been reported in literature are the genitourinary system and the central nervous system. ${ }^{[4]}$ Since the location of the disease in the body is diverse, its presentation is also varied and signs and symptoms are determined by the size and localization of the tumor. ${ }^{[3]}$

\section{Case Reports}

Case 1: A 17 years old boy with forehead swelling and fever of 2 months duration. His complete blood count (CBC) revealed leucocytosis with $10 \%$ blasts. Subsequently a bone marrow aspiration examination was performed and a final diagnosis of AML (? M3/M2) was made.

Case 2: A 30 years old lady with multiple swellings in neck since 15 days with fever. Her CBC revealed marked leucocytosis with left shift and basophilia. On bone marrow aspiration examination diagnosis of CML-accelerated phase was made.

Case 3: A 36 years old male presented with swelling in chest wall and axillary region along with weight loss since 2 months. CBC showed leucocytosis with $66 \%$ blasts. He was diagnosed as AML-M4 on bone marrow aspiration examination.

Case 4: A 39 year lady with a left side neck swelling of 20 days duration. She was anemic with $\mathrm{Hb}-3.4 \mathrm{gm} \%$ and peripheral blood examination showed $55 \%$ blasts. Further, on bone marrow aspiration she was diagnosed as AML.

The clinical details, $\mathrm{CBC}$ and bone marrow aspiration findings are summarized in the tables below (Table 1,2).

FNAC was performed for all the cases; the smears from all the four cases had an identical morphology; were cellular and revealed numerous abnormal large cells with scant to moderate cytoplasm, some cells showed cytoplasmic granules. The nuclear-cytoplasmic ratio was increased with prominent nuclear infoldings and small nucleoli. The morphology resembled that of myeloid precursors [Figure 1a \& 1b]. Mitotic figures, band forms, eosinophils, lymphocytes and fibroblasts were also seen. Hence, a diagnosis of granulocytic sarcoma was made for all the 
four cases. The peripheral blood showed myeloblast with auer rods [Figure 1c]. The peripheral smear and bone marrow aspiration findings were in concordant with the FNAC diagnosis.

Table 1: Clinical Findings .

\begin{tabular}{|l|c|c|c|c|}
\hline & CASE-1 & CASE-2 & CASE-3 & CASE-4 \\
\hline Age (yrs) & 17 & 30 & 36 & 39 \\
\hline Sex & $\mathrm{M}$ & $\mathrm{F}$ & $\mathrm{M}$ & F \\
\hline $\begin{array}{l}\text { Chief } \\
\text { Complaints }\end{array}$ & $\begin{array}{c}\text { Rt.forehead } \\
\text { Swelling x 2 months }\end{array}$ & $\begin{array}{c}\text { Multiple swelling in } \\
\text { neck x 15days }\end{array}$ & $\begin{array}{c}\text { Swelling in chest wall \& } \\
\text { axillary region x 2 months }\end{array}$ & $\begin{array}{c}\text { Swelling left side neck } \\
\text { x 20 days }\end{array}$ \\
\hline Associated Symptoms & Fever & Fever & Wt loss & - \\
\hline $\begin{array}{l}\text { Local } \\
\text { Examination }\end{array}$ & Fixed,non tender & Mobile ,nontender & Firm to hard fixed mass & Fixed,non tender \\
\hline Lymphadenopathy & + & + & + & + \\
\hline Hepatomegaly & + & + & - & - \\
\hline Splenomegaly & - & + & & \\
\hline
\end{tabular}

Table 2: Haematological Investigations

\begin{tabular}{|c|c|c|c|c|}
\hline & CASE 1 & CASE 2 & CASE 3 & CASE 4 \\
\hline $\mathrm{Hb}(\mathrm{gm} \%)$ & 5.8 & 8.2 & 8.4 & 3.4 \\
\hline TLC(cells/cumm) & 15,600 & 110,000 & 57,5000 & 12,000 \\
\hline Platelet (cells/cumm) & 24,000 & $2,00,000$ & $1,0,5000$ & $2,12,000$ \\
\hline \multicolumn{5}{|l|}{ Differential count } \\
\hline Basophils & - & 10 & - & - \\
\hline Bands & 09 & 15 & - & - \\
\hline Metamyelocytes & 05 & 10 & - & - \\
\hline Myelocytes & 11 & 15 & 02 & - \\
\hline Promyelocytes & 15 & 01 & - & \\
\hline Blasts & 10 & 06 & 66 & 55 \\
\hline Bone marrow aspiration report & AML-?M3/M2 & CML-accelerated Phase & AML-M4 & AML \\
\hline
\end{tabular}

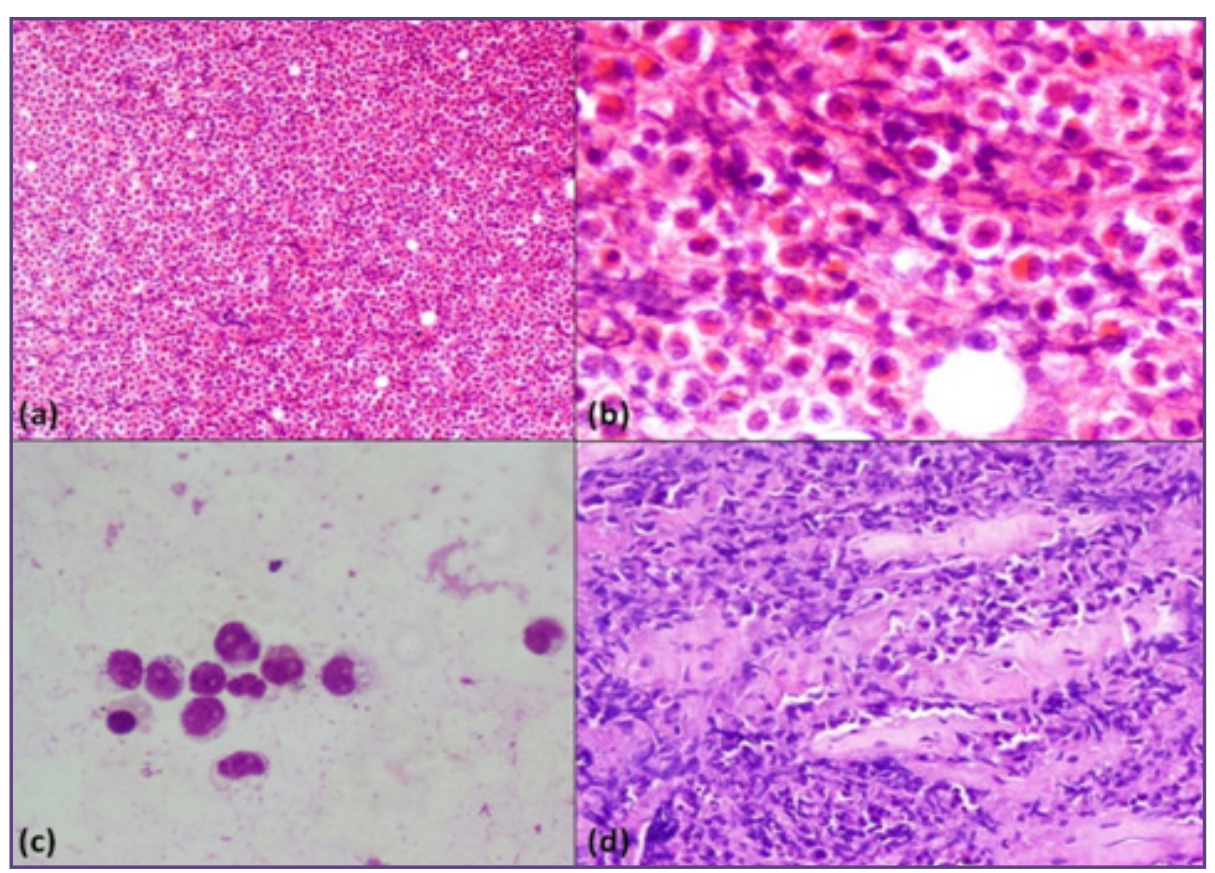

Fig. 1: (a) FNA smear: Aspirate from swelling showing a cellular smear, myeloid precursors with nuclear infoldings (PAP $X$ 10). (b) High Power (PAP X 40). (c) Peripheral smear showing myeloblast with Auer rods (Leishman $X$ 40). (d) Section showing crushed cells with eosinophilic precursors \& extensive fibrosis (H : E X 40). 


\section{Discussion}

GS was first described by Burns in 1811.The term "chloroma" was given to the lesion by King in 1853 because the tumor displays a greenish colour on exposure of the enzyme myeloperoxidase, which is high in immature tumor cells under UV light. ${ }^{[5]}$ In 1966, Rappaport was the first to name it " granulocytic sarcoma" and defined it as the " formation of invasive and destructive tumor masses composed of immature cells of granulocytic series". ${ }^{[6]}$

There is a slight male preponderance for GS, ${ }^{[2]}$ while 2 of our patients were male and 2 were female. The mean age at diagnosis as reported by Khateeb et al. was 44.4 years while our cases are falling in the age group of 15-40 years. ${ }^{[7]}$

Granulocytic sarcoma can occur in a variety of locations throughout the body with no predilection for a specific organ, ${ }^{[2]}$ in the cases reported here no specific pattern of organ involvement was observed.GS is a rare manifestation of AML which usually arises during or after the course of the disease, about $8 \%$ of patients are diagnosed on autopsy. On the other hand GS can be the first and the only manifestation of AML, creating a diagnostic challenge for the condition. ${ }^{[8]}$ The higher rate of misdiagnosis is probably a reflection of the rarity of this lesions and low index of suspicion. The association of myeloid sarcoma in patients with CML is very rare i.e. $2-4 \%$ only. ${ }^{[9]}$ One in the above mentioned case was associated with CML. The rate of misdiagnosis is $75 \%$ in a historical study, and the most frequent misdiagnosis is large cell lymphoma. In recent studies, the rate of misdiagnosis has been found to be lower with a range of $25-47 \%$. The patients were mostly mistaken for malignant lymphoproliferative disorders. The misdiagnoses are non-Hodgkin lymphoma, histiocytic lymphoma, thymoma, myeloma, eosinophilic sarcoma, extramedullary hematopiesis, mucosa associated lymphoid tissue, Ewing sarcoma and rhabdomyosarcoma , and they could not be corrected until acute leukemia was suspected by bone marrow aspiration and biopsy or peripheral blood smears. ${ }^{[10]}$ The third case we reported was also diagnosed as rhabdomyosarcoma at another hospital following which the patient was referred to our hospital for further management.

The peripheral smear and subsequent bone marrow examination are necessary to confirm the diagnosis in almost all the cases, except a few cases where extramedullary involvement precedes the leukemic presentation. In these cases, cytological preparations give us better morphological detail of blasts; the identification of Auer rods and MPO stain can help clinch the diagnosis of an undetected AML. Our case report demonstrates the clinical utility and accuracy of FNAC in making the diagnosis of MS from an extramedullary site. The visualization of an Auer rod is possible only in cytological material, so it would be much easier to make diagnoses based on FNAC and imprint smears rather than in histological sections which shows striking fibrosis [Figure 1d]. The work-up of these masses can be aided by the use of cytochemistry (MPO, Sudan Black, non-specific esterase) and immunophenotyping.

The correct diagnosis of MS is a must for adequate therapy. The treatment is similar to that for AML, even in cases of isolated tumors with no blood or bone marrow involvement. Radiotherapy has been proposed in association with chemotherapy for patients with massive tumors. ${ }^{[11]}$ In patients with AML, the progression of myeloid sarcoma has the same prognosis as the underlying leukemia. The patients with an AML associated with $\mathrm{t}$ $(8 ; 21)$ and presenting with myeloid sarcoma have a low rate of complete remission, and overall survival is poor. This appears to be in contrast to the better prognosis generally seen in AML with $\mathrm{t}(8 ; 21)$. In patients with chronic myeloproliferative and myelodysplastic syndromes, myeloid sarcoma defines a blastic transformation often associated with a short survival. ${ }^{[12]}$ Therefore we state that the present cases were cytologically evaluated and interpreted as MS. The correct recognition of this entity and differentiating it from other mimics are crucial to proper patient management and appropriate follow-up.

\section{Conclusion}

GS is a rare manifestation of AML and regarded as a "great imitator". Due to its protean manifestations and broad target population, a high index of suspicion is necessary to correctly identify this entity.FNAC can serve a useful role in identifying the morphologic characteristics of blasts and cells of myeloid lineage.Possibility of GS should therefore be considered in the differential diagnosis of poorly differentiated neoplasms.

\section{Acknowledgements}

We thank the Medical Superintendent and HOD of pathology department of KMC, Manipal for allowing us to publish this case report. All authors read and approved the final manuscript. No conflict of interest. No fund received.

\section{References}

1. Pileri SA, Ascani S, Cox MC et al. Myeloid sarcoma: clinico-pathologic, phenotypic and cytogenetic analysis of 92 adult patients. Leukemia 2007; 21: 340-350.

2. Neiman RS, Barcos M, Berard C et al. Granulocytic sarcoma: a clinicopathologic study of 61 biopsied cases. Cancer 1981; 48: 1426-1437. 
3. Bakst RL, Tallman MS, Douer D, Yahalom J. How I treat extramedullary acute myeloid leukemia. Blood 2011; 118: 3785-3793.

4. Tsimberidou AM, Kantarjian HM, Estey E et al. Outcome in patients with nonleukemic granulocytic sarcoma treated with chemotherapy with or without radiotherapy. Leukemia 2003; 17: 1100-1103.

5. Pulsoni A, Falcucci P, Anghel G et al. Isolated granulocytic sarcoma of the skin in an elderly patient: good response to treatment with local radiotherapy and low-dose methotrexate. JEurAcadDermatolVenereol. 2000; 14(3):216-218.

6. Cheah KL, Lim LC, Teong HH, Chua SH. A case of generalised cutaneous granulocytic sarcoma in an elderly patient with myelodysplastic syndrome. Singapore Med J.2002; 43(10):527-529.

7. Hani Al-Khateeb, Ahmed Badheeb, Husam Haddad et al. Myeloid Sarcoma: Clinicopathologic, Cytogenetic, and Outcome Analysis of 21 Adult Patients. Leukemia Research and TreatmentVolume 2011; Article ID 523168.
8. Messager M, Amielh D, Chevallier C, Mariette C. Isolatedgranulocytic sarcoma of the pancreas: a tricky diagnostic for primary pancreatic extramedullary acute myeloid leukemia. World J Surg Oncol. 2012; 10:13.

9. Bothale AK, Wilkinson A, Mahore SD, Patrikar AD, Bothale A; Extramedullary Granulocytic sarcoma as an initial presenting feature of chronic myeloid leukaemia. JRCP.2013; 3: 64-66.

10. Yilmaz AF, Saydam G, Fahri S, Baran Y;Review articleGranulocytic sarcoma:a systemic review. Am J Blood Res 2013;3(4):265-270.

11. Imrie KR, Kovacs MJ, Selby D et al. Isolated chloroma: The effect of early anti leukemic therapy. AnnIntern Med $1995 ; 123: 35: 1-3$.

12. Byrd JC, Edenfield WJ, Shields DJ, Dawson NA. Extramedullary myeloid Cell tumors in acute non lymphocytic leukemia: A clinical review. J Clin Oncol 1995; 13:1800-16.

*Corresponding author:

Dr Sunaina Agarwal, Govardan 9/E,Bishop rocky street,Behind vivekanand hospital Lucknow-226024 India

Phone: +919628067538

Email: sunaina.agarwal@ymail.com

Financial or other Competing Interests: None. 